\title{
Gastric malignant melanoma
}

\author{
Goundappa Loganathan • Pradyumn Singh
}

Published online: 4 August 2013

(C) Indian Society of Gastroenterology 2013

Malignant melanoma of the gastrointestinal (GI) tract is commonly metastatic, presenting along with the primary tumor or, years later, with abdominal pain, dysphagia, small bowel obstruction, or upper GI bleeding [1]. The endoscopic picture in Fig. 1, observed in a middle-aged male with a 1day history of intensely severe abdominal pain and inability to eat, depicts multiple black pigmented elevated 3-10-mmdiameter lesions with normal overlying mucosa in the fundus of the stomach. Similar lesions were noted in the lower esophagus. The patient did not have evidence of a primary melanoma, elsewhere. The diagnosis of melanoma was con- firmed by histology showing a malignant tumor composed of dyscohesive polygonal cells with pleomorphic nuclei, macronucleoli, and cytoplasmic coarse dark brown pigment (Fig. 1a, c) and by immunohistochemistry confirming staining for melan-A (Fig. 1b). Rarely anthracotic lesions in the esophagus may endoscopically mimic malignant melanoma [2]. Although primary melanoma of the stomach and esophagus is described, the multiple lesions suggested a secondary tumor with occult primary. Management may include resection, chemotherapy, or immunotherapy, and the prognosis is poor.
Fig. 1 Left upper panel shows multiple black, elevated lesions in the fundus of the stomach. a Light microscopic appearance of the tumor, hematoxylin and eosin (H\&E) stain. b Stain for melan-A positive in tumor cells. c Higher power $\mathrm{H} \& \mathrm{E}$ view showing coarse dark brown melanin pigment in tumor cells
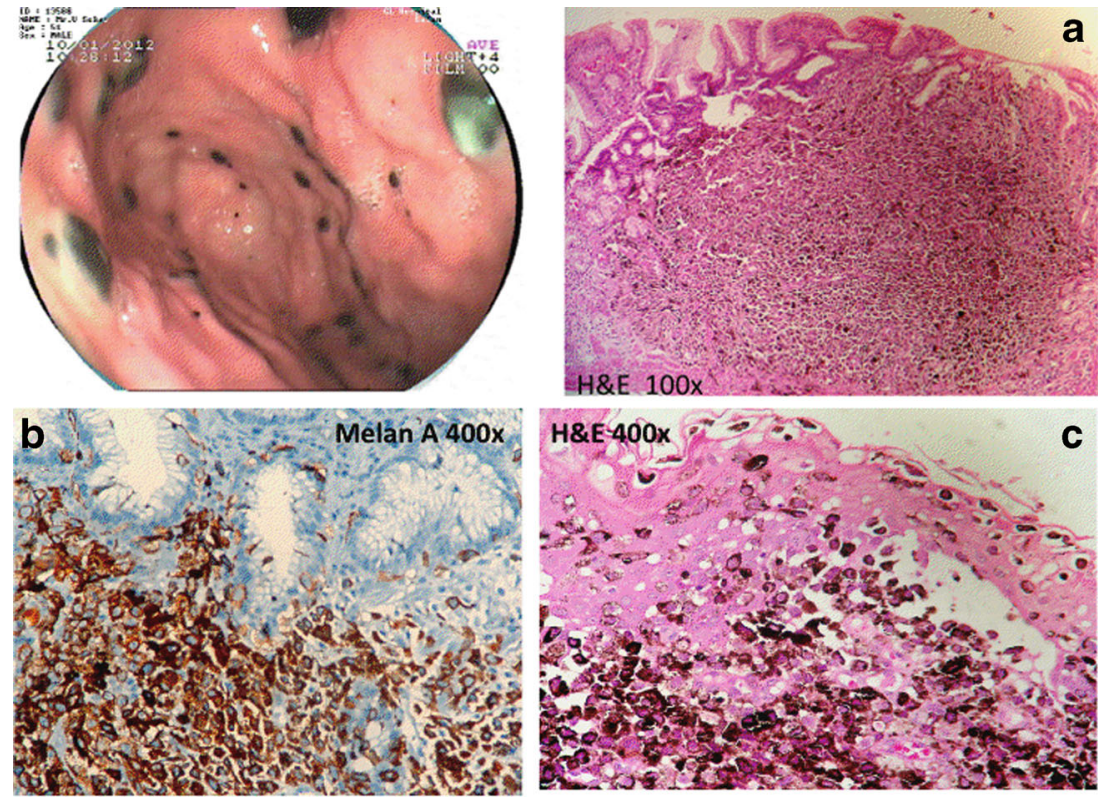

G. Loganathan $(\bowtie)$

G L Hospital, Kalaimagal Street, Swarnapuri, Salem 636 004, India e-mail: loganmanoj@gmail.com

\section{P. Singh}

Department of Pathology, Christian Medical College Hospital, Vellore 632 004, India

\section{References}

1. Liang KV, Sanderson SO, Nowakowski GS, Arora AS. Metastatic malignant melanoma of the gastrointestinal tract. Mayo Clin Proc. 2006;81:511-6.

2. Murata T, Imai T, Hoshino K, et al. Esophageal anthracosis: lesion mimicking malignant melanoma. Pathol Int. 2002;52:488-91. 